\title{
Breastfeeding information seeking behaviour among parents in Mbeya City, Tanzania
}

NEEMA MWAISELA and KELEFA MWANTIMWA

University of Dar es Salaam, P.O. Box 35092, Dar es Salaam, Tanzania

\begin{abstract}
Background: Majority of mothers in Tanzania fail to practice effective breastfeeding for various reasons. The objective of this study was to determine breastfeeding- related information seeking behaviour among parents in Mbeya City of Tanzania.

Methods: A descriptive cross-sectional survey design was used. Parents of infants and children aged between 0-2 years and health care workers were interviewed. Secondary data was collected by using documentary review. Information sought was related to the perceptions of parents towards the usefulness of breastfeeding and breastfeeding practices. The observation method was used to identify and verify the actual available, accessible, and usable breastfeeding information.

Results: The key findings suggest that information needs of parents and sources and channels used vary and depended on the time intended to breastfed the child. Mainly, the parents' needs information on nutritious food, introduction of complimentary food, breast care, and care techniques for when away from babies, frequency and implications of breastfeeding. Friends, relatives, neighbours, health professional were the most commonly sources of breastfeeding information. The majority of parents perceived that access to and usage of breastfeeding information improve breastfeeding rate, decision making, increase awareness and knowledge, and breastfeeding practices.

Conclusion: Information seeking behaviour varies from one parent to another hence the varying preferences in informal and formal sources. Increasing accessibility and usability of breastfeeding information is necessary in ensuring that one of the basic human rights is safeguarded
\end{abstract}

Keywords: Breastfeeding, information, seeking behaviour, Tanzania

\section{Introduction}

Access to and use of health information provides parents with knowledge on health care of infants and children, preventing diseases, and dealing with breastfeeding difficulties. It appears that well informed parents know the health benefit of breastfeeding, the preferred duration for exclusive breastfeeding, when to provide supplementary food, nutrition and health benefit of breastfeeding (Jiang et al., 2012; Davis et al., 2016). Likewise, a study by Victora et al. (2016) underlines that parents need information about breastfeeding to be aware of matters such as breast care, duration of feeding, frequency of feeding, and proper eating for mothers.

The breastfeeding information is presented through different channels and in different formats. In general, information sources can be categorized as electronic, print, and oral (Carolan, 2007; Shieh et al., 2009). In fact, pamphlets and leaflets, health practitioners and knowledgeable people in the communities (WHO, 2015) are commonly used to disseminate breastfeeding information. For example, parents may have access to breastfeeding information from health care workers and mothers (Petit, 2010; WHO, 2015). Leach \& Kilama (2009) confirmed that the main sources of information for mothers are their family members rather than health care practitioners. On top of that, electronic sources are becoming important and popular for disseminating health information among health products consumers and pregnant women (Hillyer et al., 2017) which are preferred due to the ease of access to information it provides (Clarke et al., 2015). Breastfeeding decisions and practices can be influenced by various factors such as culture, attitudes, perceptions, and societal differences in both developing and developed countries (Shirima et al., 2001; Bandyopadhway, 2009; Wanjohi et al., 2017).

Despite the concerted initiatives made by the governments, organizations and health professionals to promote breastfeeding information, poor breastfeeding practices have been cited as one of the reasons for unhealthy children globally (Hashim et al., 2017; Mbwana, 2012; 
Edmond et al., 2006). These suggest that poor breastfeeding continues to be a challenge in many countries globally. It has been revealed that about $80 \%$ of children are not optimally breastfed leading to different health problems (UNICEF, 2016). The overall rate of breastfeeding for children in sub-Saharan Africa is still low (Azuine et al., 2015). This situation is attributed to parents' reliance on breastfeeding information received from informal sources such as family members and friends (Sim et al., 2014; Leach \& Kilama, 2009). Accordingly, the trend is associated with limited access to breastfeeding information among parents (Fornasaro-Donahue, 2012). It is worth noting that majority of parents lack access to quality information which would help them in decision making on breastfeeding as a result children are put at a risk of having health issues (Jiang et al., 2012).

The problem of limited access to information and knowledge appears to be influenced by cultural, economic, education, and social factors (Shirima et al., 2001). Evidently, majority of mothers in Tanzania fail to practice effective breastfeeding as recommended (Hashim et al., 2017). The main attributing factors for poor breastfeeding include low knowledge on its importance (Cai et al., 2012), negative attitudes, norms, values and other traditions about breastfeeding among parents (Weaver \& Njeri, 2010). Most studies on breastfeeding practices in Tanzania have focused on factors affecting breastfeeding among mothers and malnutrition (Saka, 2012; Mbwana et al., 2013, Maonga et al., 2016). However, the role of information in breastfeeding has received little attention. There is dearth of information on breastfeeding information needs or preferred information sources, channels, and challenges faced in accessing and utilizing such information in Tanzania. It is against this background that this study aimed to determine the access to and use of breastfeeding information in Mbeya City, Tanzania. The specific objectives were threefold: to identify breastfeeding information needs among parents; examine breastfeeding information sources and channels used by parents; and explore the perceptions of parents towards the usefulness of breastfeeding information on breastfeeding practices.

\section{Materials and Methods}

\section{Study area and design}

The study was conducted in Mbeya City located in the south-western highlands of Tanzania. Mbeya City was selected due to the fact that it is among the areas having high rate of low breastfeeding practices and unhealthy children (UNICEF, 2011). In this study, descriptive crosssectional survey design was used to determine breastfeeding information seeking behaviour among parents. The study population was made of parents of infants and children aged between 0-2 years and health care workers from two health centres, namely lgawilo and Ruanda. Parents of infants/children between 0-2 years who attending the health centres and health care workers were included in the study. Whereas, purposive sampling was used to select health care workers, simple random sampling was used to obtain parents of infants/children.

\section{Data collection}

Secondary data was collected by using documentary review while questionnaire, interview, observation methods were used to collect primary data. The study obtained secondary data by reviewing different reports, published and unpublished paper. Primary data was gathered by using questionnaires, an interview guide and participant observation guide. There were different kinds of questions such as multiple and fill in with nominal, ordinal and Likert's scale questions. Semi-structured interviews were used to collect data from health care workers. This was done purposively to obtain information which would not have been easily obtained through questionnaires. The study used the observation method to identify and verify the actual available, accessible, and usable breastfeeding information facilities. 


\section{Data analysis}

Both qualitative and quantitative data were organized, described, coded, analysed, and generated. The study used Statistical Package and Service Solution (SPSS) Software (version 20) to generate percentages and frequencies from quantitative data. In all, descriptive (i.e. frequencies and percentages) and inferential (Chi-Square test, regression and correlations) statistics were performed to measure trends, differences, level of association, direction of association and strength. Content and thematic analysis was used to analyse qualitative data.

\section{Results}

\section{Background information of the respondents}

A total of 70 parents of infants and children aged between 0-2 years participated in the study. The majority $(92.9 \%)$ of parents were females ( $p$-value $=0.000)$. The majority $(57.1 \%)$ of respondents were aged $25-36$ years $(p$-value $=0.000)$. About two thirds $(65.7 \%)$ of the respondents were married. About half $(47.1 \%)$ of respondents had monthly incomes of less than US\$25. Forty percent of respondents had primary level of education (Table 1).

Table 1: Background information of the respondents $(n=70)$

\begin{tabular}{|c|c|c|c|c|}
\hline Variable & Response & Frequency & Percentage & P-value \\
\hline \multirow[t]{2}{*}{ Health Centre } & Ruanda & 39 & 55.7 & 0.339 \\
\hline & Igawilo & 31 & 44 & \\
\hline \multirow[t]{2}{*}{ Sex } & Female & 65 & 92.9 & 0.000 \\
\hline & Male & 5 & 7.1 & \\
\hline \multirow[t]{2}{*}{ Marital status } & Married & 46 & 65.7 & 0.000 \\
\hline & Not married & 24 & $34 \cdot 3$ & \\
\hline \multirow[t]{3}{*}{ Age (years) } & $15-24$ & 15 & 21.4 & 0.000 \\
\hline & $25-36$ & 40 & 57.1 & \\
\hline & $37-46$ & 15 & 21.4 & \\
\hline \multirow[t]{5}{*}{ Education } & Primary & 28 & 40.0 & 0.000 \\
\hline & Secondary & 12 & 17.1 & \\
\hline & Certificate & 14 & 20.1 & \\
\hline & Diploma & 10 & $14 \cdot 3$ & \\
\hline & University & 6 & 8.6 & \\
\hline \multirow[t]{3}{*}{ Income (in US Dollar) } & $<25$ & 33 & 47.1 & 0.045 \\
\hline & $26-50$ & 20 & 28.6 & \\
\hline & $>50$ & 17 & $24 \cdot 3$ & \\
\hline \multirow[t]{2}{*}{ No. of children } & $1-3$ & 60 & 85.7 & 0.000 \\
\hline & $4-6$ & 10 & $14 \cdot 3$ & \\
\hline \multirow[t]{3}{*}{ Breastfeeding time } & Partial & 39 & 55.7 & 0.000 \\
\hline & Exclusive & 25 & $35 \cdot 7$ & \\
\hline & Predominant & 6 & 8.6 & \\
\hline \multirow[t]{2}{*}{ Breastfeeding schedule } & Used & 10 & $14 \cdot 3$ & 0.000 \\
\hline & Not used & 60 & 85.7 & \\
\hline
\end{tabular}

More than half ( $55.7 \%$ ) of respondents intended to breastfeed their children partially (only during the early days of the children's lives. About one third (35.7\%) of the respondents said they intended to exclusively breastfeed their children (Table 1). Respondents attributed their inability to exclusively breastfeed to challenges they face. In her words, one of the parents narrated that: "Mothers have to work too and sometimes they are too busy to attend to their children. In addition, some of institutions do not give mother permission to attend to and breastfeed their children. However, sometimes failure to exclusively breastfeed is just a result of mothers' failure to consider breastfeeding as an important thing. There is a need to create awareness and educate people on the value of breastfeeding to encourage exclusive breastfeeding". These narratives confirm that a significant percentage of mothers do not effectively breastfed their children. 
Respondents were asked if they followed breastfeeding schedules. The study revealed that $60(85.7 \%)$ of the respondents did not follow any schedule when breastfeeding while only 10 (14.3\%) said to follow a particular schedule (Table 1). The respondents attributed this to factors like time constraints, cultural practices, life stress, lack of experience, and insufficiency of knowledge and information on breastfeeding. On this, one parent from Ruanda Health Centre stated that: "Although it is vital for every mother to follow a proper breastfeeding schedule, without education and enough knowledge, such a thing is not possible". Healthcare professionals disclosed that a good schedule for breastfeeding requires that a mother feeds her child every 2 to 3 hours. Statistical significant differences were found on sex, marital status, age, level of education, income, number of child, time of breastfeeding and breastfeeding schedule ( $p$-value < 0.05). Area of resident did not show any statistical difference.

\section{Breastfeeding information needs}

To determine their needs, the respondents were asked to outline the types of information they need to improve their breastfeeding practices. Sixty-three (90\%) of the respondents mentioned information about nutritional foods while 60 (85.7\%) mentioned information about when one can introduce solid food to children's diet. Fifty-eight (82.9\%) respondents said they needed to know how to introduce and select complimentary food, 56(80.0\%) wanted to know more on breast care, and 53 (75.7\%) on caring for themselves when away from their babies. Forty-seven (67.1\%) respondents wanted to know more about breastfeeding implications, $43(61.4 \%)$ had interest in knowing the right frequency for breastfeeding, 35 (50.0\%) on baby cues, while $27(38.6 \%)$ on bottle feeding (Table 2).

Table 2: Breastfeeding information need $(n=70)$

\begin{tabular}{lccccc}
\hline Breastfeeding information & \multicolumn{3}{c}{ Time intend to breastfed } & Total & P-value \\
& Exclusive & Predominant & Partial & & \\
\hline Breast care & $23(92 \%)$ & $4(66.7 \%)$ & $29(74.4 \%)$ & $56(80 \%)$ & 0.158 \\
Breastfeeding routine & $8(32 \%)$ & $1(16.7 \%)$ & $17(43.6 \%)$ & $26(37.1 \%)$ & 0.358 \\
Frequency of feeding & $23(92 \%)$ & $0(0.0 \%)$ & $20(51.3 \%)$ & $43(61.4 \%)$ & 0.000 \\
Bottle feeding & $5(20 \%)$ & $2(33.3 \%)$ & $20(51.3 \%)$ & $27(38.6 \%)$ & 0.105 \\
Starting solid food & $23(92 \%)$ & $3(50 \%)$ & $34(87.2 \%)$ & $60(85.7 \%)$ & 0.028 \\
Caring baby when away & $22(88 \%)$ & $2(33.3 \%)$ & $29(74.4 \%)$ & $53(75.7 \%)$ & 0.019 \\
Storing breast milk & $6(24 \%)$ & $2(33.3 \%)$ & $13(33.3 \%)$ & $21(30.0 \%)$ & 0.717 \\
Breastfeeding implications & $15(60 \%)$ & $3(50 \%)$ & $29(74.4 \%)$ & $47(67.1 \%)$ & 0.317 \\
Complementary food & $20(80 \%)$ & $3(50 \%)$ & $35(89.7 \%)$ & $58(82.9 \%)$ & 0.050 \\
Baby cues & $16(64 \%)$ & $0(0.0 \%)$ & $19(48.7 \%)$ & $35(50.7 \%)$ & 0.031 \\
Nutritional food & $22(88 \%)$ & $6(100 \%)$ & $35(89.7 \%)$ & $63(90 \%)$ & 0.677 \\
\hline
\end{tabular}

In all, the results signify that the information needs of the parents participated in the study vary and depend on the time intend to breastfed. The information needs on breast care, breastfeeding routine, bottle feeding, expressing and storing breast milk, breastfeeding implications and nutritional food were not significantly different ( $p$-value $>0.05$ ) between those who intended to partially, exclusively and predominantly breastfed. However, information needs on frequency of breastfeeding, starting solid food, caring baby when away, introducing complementary food, and baby cues were significantly different ( $p$-value $<0.05$ ) between those who intended to perform exclusive, partial and predominant breastfeeding (Table 2). To substantiate the findings, one female respondent said: "Breastfeeding is not a problem in our community; the problem is the availability of information on breastfeeding techniques and selection of the required food for our children". Another respondent added that: "Knowing what a child needs is very challenging. Sometimes child crying is an indication of being hungry or sick while not on other occasions. It is important to know different indications for different children's needs". 


\section{Sources and channels of breastfeeding information}

Fifty (71.4\%) of respondents admitted to having access to different breastfeeding information sources and channels while $20(28.6 \%)$ said they had not accessed the sources and channels ( $p$ value $<0.05$ ). When asked about the sources they use to access breastfeeding information, 59 $(84.3 \%)$ of the respondents cited relatives and parents, $56(80 \%)$ mentioned healthcare workers, and $40(57.1 \%)$ mentioned friends. Regarding the usability of breastfeeding information, 40 (57.1\%) of respondents had not used any breastfeeding information sources while 30 (42.9\%) admitted to use the information ( $p$-value $<0.05$ ). Thirty-four $(48.6 \%$ ) of the respondents said that they found the available sources effective while 28 (40\%) found them very effective (Table 3 ).

Regarding sources and channels of breastfeeding information, one respondent had these to say: "A significant percentage of parents rely on information provided by friends and relatives which sometimes is not reliable or enough to suffice the breastfeeding information needs. Such parents do not come to our dispensaries to get the relevant breastfeeding information". Another parents said "when I need information on child health, I prefer to consult my parents, friends, neighbours, and other relatives rather than consulting health care professionals. In our society, we depend on neighbourhoods and relationship for different kinds of health information". Apart from that, one of the parents had the following to substantiate the findings from the survey results: "Since I gave birth to my first child, I have never accessed breastfeeding information through television and radio. Usually, I have access to breastfeeding information from other sources such as text messages, friends, and relatives".

Table 3: Access, usage, sources and channels of breastfeeding information $(n=70)$

\begin{tabular}{|c|c|c|c|c|}
\hline Variable & Response & Frequency & Percentage & P-value \\
\hline \multirow[t]{2}{*}{ Access to information } & Access & 50 & 71.4 & 0.000 \\
\hline & No access & 20 & 28.6 & \\
\hline \multirow[t]{13}{*}{ Source of information } & Parents/relatives & 59 & 84.3 & 0.011 \\
\hline & Friends & 40 & 57.1 & \\
\hline & Health workers & 38 & 54.3 & \\
\hline & Flyers & 28 & 40 & \\
\hline & Television & 25 & 37.7 & \\
\hline & Radio & 24 & 34.3 & \\
\hline & Internet & 20 & 28.6 & \\
\hline & Mobile phones & 17 & $24 \cdot 3$ & \\
\hline & Banner & 14 & 20 & \\
\hline & Social media & 13 & 18.6 & \\
\hline & Magazine & 13 & 18.6 & \\
\hline & News papers & 11 & 15.7 & \\
\hline & Mobile clinic/outreach & 4 & 5.7 & \\
\hline \multirow[t]{2}{*}{ Usage of information } & Used & 30 & 43 & 0.002 \\
\hline & Not used & 40 & 57.1 & \\
\hline \multirow[t]{3}{*}{ Effectiveness of information } & Very Effective & 28 & 40.0 & 0.000 \\
\hline & Effective & 34 & 48.6 & \\
\hline & Not Effective & 8 & 11.4 & \\
\hline
\end{tabular}

Importance of breastfeeding information

More than half $(72.9 \%)$ of the respondents admitted that access to breastfeeding information helped to improve child health while $48(68.6 \%)$ said the information increases parents' awareness on child health. Further, 48 (68.6\%) respondents said access to such information helped to increase breastfeeding rate, $47(67.1 \%)$ said it helped to reduce mortality rate, and 46 $(65.7 \%)$ of them believed it helped in making decisions on breastfeeding (Table 4). One of the parents from Ruanda Health Centre said that: "The information I get from my mother, friends, and family members allows me to gain new knowledge that helps me to improve breastfeeding hence enhancing my children's health. This information is very important when it comes to my children's 
health". Likewise, the role of information in breastfeeding was apparent as one respondent explained that: "The doctor saved my life. I went to a health centre where they explained the importance of exclusive breastfeeding. I did it, and today I have no regret and I am proud of my little girl.

Table 4: Perceived importance of breastfeeding information

\begin{tabular}{lcccc}
\hline Perception $(\mathbf{n}=\mathbf{7 0})$ & \multicolumn{2}{c}{ Agree } & \multicolumn{2}{c}{ Not agree } \\
& Frequency & Percent & Frequency & Percent \\
\hline Improves child health & 51 & 72.9 & 2 & 2.8 \\
Increase awareness & 48 & 68.6 & 1 & 1.4 \\
Reduce mortality rate & 48 & 67.1 & 6 & 8.6 \\
Helps in decision making & 46 & 65.7 & 5 & 7.1 \\
Increase breastfeeding rate & 48 & 68 & 9 & 12.9 \\
\hline
\end{tabular}

\section{Discussion}

Basing on the findings, the information needs of the parents vary and depend on the time intended to breastfeed. Basically, the parents need information on nutritious food, caring techniques, frequency and implications of breastfeeding. The findings indicate that majority of respondents cited nutritional food, time for starting giving solid food, introduction of complimentary food, breast care, and care techniques when away from babies as the most important information on breastfeeding. These results are an indication that majority of parents in the study area have multiple information needs. In addition to expressing the information needs, the need to satisfy them was evidently critical. All in all, the results show that parents in Mbeya City know the information they need but the absence of such information has left their knowledge gaps unfilled. These findings are in line with those from Victora et al. (2016). In contrast, Jiang et al. (2012) reported that parents are not aware of their need for information and knowledge on breastfeeding and as a result, their decision making on such practices is not good enough.

In addition to that, the parents in this study expressed their need for information on the right daily breastfeeding frequency and its implications on mothers and children. Similar findings have been observed by Jiang et al. (2012) in China. The study also found that noticeable percentages of parents need information on bottle feeding, breastfeeding routine, and expressing and storing milk. It is unfortunate that despite their importance, these kinds of information were not prioritized by the majority of parents. In contrast, previous studies (Victora et al., 2016; WHO, 2015) reported that expressing and storing milk, breastfeeding routine, and bottle feeding as important areas which parents needed to be well informed about.

Apart from that, majority of the parents participated in the study had access to different breastfeeding information. These findings are not in harmony with what one would assume to be the reality on the ground where respondents' interaction with health professionals is generally expected to provide them with information (Leach \& Kilama, 2009; Petit, 2010). This discrepancy is further highlighted by the observation of the presence of sessions on child health and development during the parents' clinic visits. In real sense, the parents who participated in this study had direct and indirect access to breastfeeding information. Further, the findings suggest that parents are aware of and have opportunities to access breastfeeding information through electronic sources, oral and print sources.

These results reveal the impact social and cultural relationships, and neighbourhoods have access to information on breastfeeding. This reliance on such sources can be attributed to costs, lack of awareness on credible sources, insufficiency of knowledge and experience needed to use other sources, beliefs, level of education, and income. The parents are likely to use cheaper informal sources of information to avoid costs associated with formal ones. Similarly, 
health care workers were found to be one of the most used sources of breastfeeding information due to the fact that most parents attend clinics with their children where information sharing takes place. In contrast, Mbwana et al. (2013) found that Tanzanian health facilities do not provide routine seminars and workshops on child health hence parents had to depend on friends, neighbours, and relatives as main sources of breastfeeding information. In a study in Uganda, Petit (2010) found that two-thirds of parents receive breastfeeding information from different informal sources. Respondents also confirmed that apart from the costs associated with some mass media types, time constraint was a barrier to accessing to the information through mass media. The findings concur with those from other studies (Shirima et al., 2001). A study in Kenya reported that mobile phones are an important source for health communication in remote areas (Kazi et al., 2017).

The study found that a significant percent of respondents perceived access to and use of breastfeeding information as important in improving breastfeeding practices hence improving child health. Through such information some of the parents were aware that breastfeeding increases vitamins, proteins, iron, and other nutrients which improve child health. The findings further reveal that the majority of parents in the area believed that access to breastfeeding information increases awareness on breastfeeding practices. This indicates that with the help of proper information, parents become aware of the importance of proper breastfeeding in promoting healthy growth and development of the children. These findings also reveal that parents believe that access to and use of breastfeeding information enhances decision making (WHO, 2015). Access to and use of breastfeeding information was also credited with improving breastfeeding rate. This is linked to the fact that the knowledge gained from the information helps parents to become aware of and well informed about the benefits of breastfeeding to children, mothers and the society.

The findings of this study attest that parents have various breastfeeding information needs to be fulfilled. In other words, the results indicate that information seeking behaviour varies from one parent to another hence the varying preferences in informal and formal sources. In particular, the findings have revealed that informal sources such as friends, relatives, neighbours, health professional are the most commonly used in the study area. Increasing accessibility and usability of breastfeeding information is necessary in ensuring that one of the basic human rights is safeguarded. Therefore, deliberate efforts to enhance access to and use of breastfeeding information must be put in place in order to improve breastfeeding practices which in turn will directly or indirectly improve child and mother health.

\section{References}

Azuine, R.E., Murray, J., Alsafi, N. \& Singh, G.K. (2015) Exclusive Breastfeeding and under-five mortality, 2006-2014: A cross-sectional analysis of 57 low-and middle income countries. International Journal of MCH and AIDS 4: 13-21.

Bandyopadhway, M. (2009) Impact of ritual pollution on lactation and breastfeeding practices in rural West Bengal, India. International Breastfeeding Journal 4 (2).

Cai, X., Wardlaw, T., \& Brown, D.W. (2012) Global trends in exclusive breastfeeding. International Breastfeeding Journal 7: 1-5.

Carolan, M. (2007) Health literacy and the information needs and dilemmas of first-time mothers over 35 years. Journal of Clinical Nursing 16: 1162-1172.

Clarke, M.A., Moore, J.L., Steege., L.M., Koopman, R.J., Belde, J.L., Canfield, S.M., Meadows, S.E., Elliott, S.G. \& Kim, M.S. (2015) Health information needs, sources, and barriers of primary care patients to achieve patient-centered care: A literature review. Health Informatics Journal 22: 992-1016. 
Davis, R.E., Cole, S.M., McKenney-Shubert, S.J., Jones, S.J. \& Peterson, K.E. (2017) An exploration of how Mexican American WIC mothers obtain information about behaviors associated with childhood obesity risk. Journal of Nutrition Education and Behavior 49: 187-195.

Edmond, K.M., Zandoh, C., Quigley M, A., Amenga-Etego, S, Owusu-Agyel, S. \& Kirkwood, B.R. (2006) Delayed breastfeeding initiation increases risk of neonatal mortality. Pediatrics Journal 117: 380-386.

Fornasaro-Donahue, V. (2012) Increasing breastfeeding in WIC participants using information. Open Access Master's Theses 140. Retrieved 12 August 2017 from: http://digitalcommons.uri.edu/theses/140.

Hashim, T.H., Mgongo, M., Katanga, J., Uriyo, J.G., Damian, D.J., Stray-Pedersen, B., Wandela, M., \& Msuya, S.E. (2017) Predictors of appropriate breastfeeding knowledge among pregnant women in Moshi Urban, Tanzania: a cross-sectional study. International Breastfeeding Journal 12:11.

Hillyer, G.C., Karen, M., Schmitt, Lizardo, M., Reyes, A., Bazan, M., Alvarez, M.C., Sandoval, R., Abdul, K. \& Orjuela, M.A. (2017) Eletronic communication channel use and health information sources among Latinos in Northern Manhattan. Journal Community Health 42: 349-357.

Jiang, H., Li, M., Yang, D., Wen, L.M., Hunter, C., Gengsheng, H., \& Qian, Xu. (2012) Awareness, intention and needs regarding breastfeeding: findings from first mothers in Shangai, China. Breastfeeding Medicine 7: 526-534.

Leach, V. \& Kilama, B. (2009) Preventing malnutrition in Tanzania: a focused strategy to improve nutrition in young children. REPOA Brief No.15. Dar es Salaam, Tanzania.

Maonga, A., Mahande, M., Damian, D. \& Msuya, S. (2016) Factors affecting exclusive breastfeeding among women in Muheza, Tanga. Journal of Maternal Child Health 20: 77-87.

Mbwana, H. (2012) Exclusive Breastfeeding: Mothers' Awareness and Health Care Providers' Practices during Antenatal Visits in Morogoro, Tanzania. PhD Thesis, Massey University, New Zealand). Retrieved from http://hdj.handle.net/10179/3863.

Mwantimwa, K. (2012) The use of pull information mode to support poverty reduction programmes in rural Tanzania: A case of Monduli and Bagamoyo districts. Universiteit Antwerpen (Belgium), ProQuest Dissertations Publishing 3571502.

Petit, A. (2010) Perception and knowledge on exclusive breastfeeding among women attending antenatal and postnatal clinics: A study from Mbarara Hospital, Uganda. Tanzania Medical Students Association Journal 16: 27-30.

Saka, J. (2012) Factors Influencing Exclusive Breastfeeding among HIV Positive Mothers at Ilala Municipality Dare es Salaam. Master Dissertation, Muhimbili University of Health and Allied Sciences, Tanzania.

Sayakhot, P. \& Carolan-Olah, M. (2016) Internet use by pregnant women seeking pregnancyrelated information: a systematic review. BMC Pregnancy and Childbirth 16:65.

Scott, J.A., Shaker, M. \& Reid, B. (2004) Parental attitude towards Breastfeeding: their association with feeding outcome at hospital discharge. Birth 3: 125-131.

Shieh, C., Broome, M.E. \& Stump, T.E. (2009) Factors associated with health information-seeking in low income pregnant women. Women Health 50: 426-442.

Shirima, R., Medhin, G. \& Greiner, T. (2001) Information and socio economic factors associated with early breastfeeding practices in rural and urban Morogoro, Tanzania. Acta Pcédiatrica Journal 90: 936-942.

Sim, T.F., Hattingh, H.L., Sherriff, J., Tee, L.B.G. (2014) Perspectives and attitudes of breastfeeding women using herbal galactagogues during breastfeeding: a qualitative study. BMC Complementary and Alternative Medicine Journal 14: 1-11.

UNICEF (2011) Levels and trends in child mortality. Retrieved 2 September, 2017 from: http://www.childmortality.org/files_v20/download/Levels\%20and\%20Trends\%20in\%20Child\%2 oMortality\%20Report\%202011.pdf 
Victora, C.G., Bahl, R., Barros, A.J.D., Franca, G.V.A., Horton, S., Krasevec, J., Murch, S., Sankar, M, J., Walker, N. \& Rollins, N.C. (2016) Breastfeeding in the $21^{\text {st }}$ century: epidemiology, mechanisms, and lifelong effect. Lancet 387(10017): 475-490.

Wanjohi, M., Griffiths, P., Wekesah, F., Muriuki, P., Muhia, N., Musoke, R.N., Fouts, H.N., Madise, N.J. \& Kimani-Murage, E.W. (2017) Sociocultural factors influencing breastfeeding practices in two slums in Nairobi, Kenya. International Breastfeeding Journal 12: 5 .

Weaver, B., \& Njeri, V. (2010) Health information-seeking behaviours, health indicators, and health risks. Journal of Public Health 100: 1520-1525.

WHO (2015) World Health Statistics. Part Il Global Indicators. World Health Organization. Retrieved from: http://www.who.int/glo/publications/world health statistics/EN-WH 